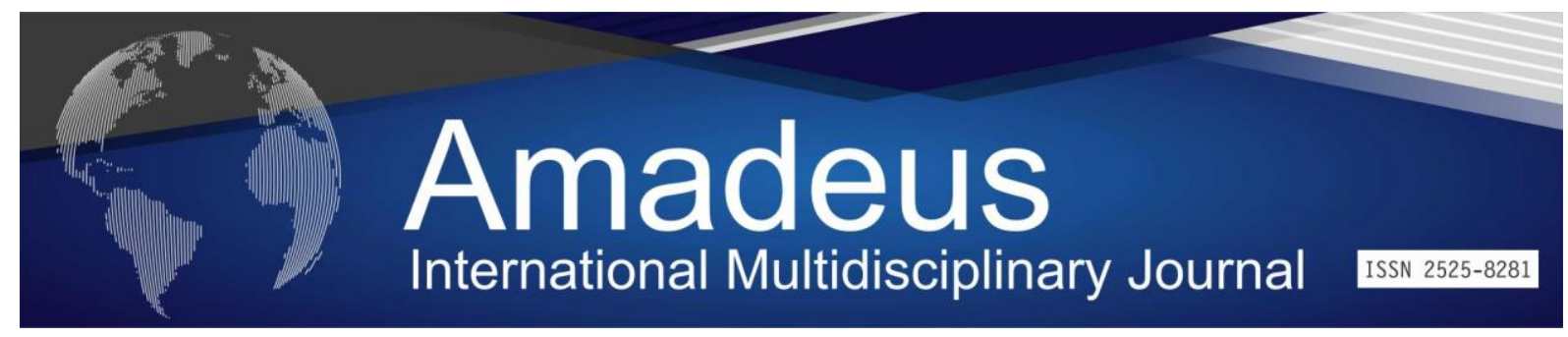

DOI: 10.14295/aimj.v5i9.141

\title{
Current Approach to Acute Apendicitis: A Case Report
}

George Wallisson Severo de Sá1,

Hermes Melo

Teixeira Batista ${ }^{2}$, Francisco Felippe

Rolim $^{3}$
Abstract: Acute appendicitis is one of the most common surgical emergencies. It may have complications such as abscess, rupture, phlegmon. It affects more men. The diagnosis is eminently clinical and complementary exams may be used for doubtful or complicated cases. Abdominal computed tomography is the main complementary exam. As for treatment, there are three approaches, the first conservative with antibiotics; the second, which consists of appendectomy; and the third, which consists of initial conservative treatment, the second with interval appendectomy. Laparoscopic appendectomy has stood out with greater emphasis on treating complicated appendicitis due to better postoperative outcomes and lower complication rate.

Keywords: 1. Appendicitis; 2. Laparoscopy; 3.Interval Appendicectomy 4. Phlegmon.

\section{Abordagem Atual da Apendicite Aguda: Um Relato de Caso}

Resumo: A apendicite aguda é uma das emergências cirúrgicas mais comuns. Pode cursar com complicações como abscesso, rotura, flegmão, acometendo mais o sexo masculino. O diagnóstico é eminentemente clínico, podendo utilizar-se de exames complementares para casos duvidosos ou complicados. A tomografia computadorizada de abdome é o principal exame complementar. Quanto ao tratamento, há três abordagens, a primeira, conservadora com antibióticos; a segunda, que consiste na apendicectomia; e a terceira, que consiste no tratamento conservador inicial, seguida de apendicectomia com intervalo. A apendicectomia laparoscópica tem se destacado com maior ênfase no tratamento de apendicite complicada em decorrência de melhores resultados no pósoperatório e menores taxas de complicações.

Palavas-chave: 1.Apendicite; 2. Laparoscopia; 3.Apendidectomia com intervalo; 4. Flegmão

\footnotetext{
${ }^{1}$ Resident in Surgery at Cariri Regional Hospital. Juazeiro do Norte, Ceará, Brasil.

${ }^{2}$ Doctor from the Federal University of Ceará -UFC. PhD from the Faculty of Medicine of ABC. Santo André-SP. Professor in UNIFAP Juzeiro do Norte - CE, Brasil. Contact: hermes2710batista@gmail.com.

${ }^{3}$ Surgeon of the Digestive System at Cariri Regional Hospital. Juazeiro do Norte, Ceará, Brasil.
} 


\section{Introdução}

A apendicite aguda é uma das emergências cirúrgicas mais comuns. A apendicite é um dos diagnósticos cirúrgicos mais prevalentes em pacientes jovens que se apresentam ao pronto-socorro com dor abdominal aguda, perfazendo um total de aproximadamente $7 \%$. A apresentação clássica começa com dor periumbilical seguida de anorexia, náusea, vômito, dor no quadrante inferior direito (QID), com ou sem febre, e geralmente leucocitose; entretanto, esses achados não são específicos para o diagnóstico e ocorrem em pacientes com outras etiologias para a dor abdominal. A incidência geral de apendicite aguda varia entre 76 e 227 casos por 100.000 habitantes por ano em diferentes países (Talan EA et al., 2017; Cheng et al., 2017).

A apendicite aguda pode ser amplamente dividida em dois subgrupos: apendicite simples (por exemplo, apendicite precoce ou não complicada) e apendicite complicada (por exemplo, apendicite gangrenosa, perfurada e apendicite com flegmão ou abscesso). Aproximadamente $3,8 \%$ a $7 \%$ dos pacientes apresentam fases avançadas do processo inflamatório, após os mecanismos de defesa isolarem o agente inflamatório, formando um flegmão ou uma doença com abscesso periapendicular bem definido. A apendicectomia ainda consiste na principal abordagem terapêutica. (Cheng et al., 2017; Al-Kurd et al., 2018)

$\mathrm{O}$ risco de desenvolvimento da doença ao longo da vida é de 8,6\% entre indivíduos do sexo masculino e 6,7\% entre as mulheres (Talan EA et al, 2017; Gavriilidisa et al., 2019; Di Saverio et al., 2016). Apesar de envolver um maior processo inflamatório, o prognóstico para uma apendicite complicada com flegmão ou abscesso apendicular é bom. A taxa geral de mortalidade é inferior a $1 \%$. No entanto, está associada a maior morbidade do que a apendicite simples. (Cheng et al., 2017)

O diagnóstico faz-se baseado na história clínica, exame físico associado com investigações laboratoriais, além de poder ser complementado por imagens radiológicas. Estes dados podem ser usados em combinação para formar sistemas de pontuação que aumentam a probabilidade diagnóstica (Al-Kurd et al., 2018).

É notória a tentativa de mudança no tratamento da apendicite aguda, principalmente com o advento da laparoscopia, entretanto, existem evidências de que o tratamento conservador da apendicite aguda, ou seja, apenas com antibioticoterapia, tem aumentado o índice de apendicite complicada. (Gavriilidisa et al., 2019). O uso de antibióticos como primeira linha 
de tratamento demonstrou uma taxa de sucesso inicial de $73 \%$, entretanto, notou-se falha terapêutica e conversão para cirurgia em 48 horas, ocorrendo em $50 \%$ dos pacientes. Ademias, o risco de recorrência em 1 ano é de 50\%, podendo-se considerar como principal fator de risco para o desenvolvimento de apendicite complicada. (Gavriilidisa et al., 2019)

Até o momento, não há diretrizes padronizadas para o manejo da apendicite complicada. O período do intervalo cirúrgico para submeter o paciente a apendicectomia em casos de apendicite complicada não foi estabelecido, além disso, ainda é controversa a necessidade de intervalo para realizar apendicectomia caso a abordagem não operatória inicial for escolhida. (Talan EA et al., 2017; Cheng et al., 2017; Gavriilidisa et a.l, 2019; Forsyth et al., 2016).

Portanto, o objetivo do presente estudo é debater o que há de mais recente na abordagem da apendicite aguda, principalmente no tocante a apendicectomia com intervalo, discutindo suas vantagens e limitações.

\section{Estudo do Caso}

Paciente M.S.F., 76 anos, masculino, procedente de outro hospital da rede, foi admitido após queixa de dor abdominal há 30 dias da admissão. A dor caracterizada como em cólica, intermitente, iniciou de maneira progressiva, com períodos de remissão, ocupando região umbilical e posteriormente fossa ilíaca direita, de moderada intensidade, sem irradiação, referindo náusea e anorexia como fatores associados, não referindo fatores desencadeantes ou atenuantes. Porém, no exame admissional já encontrava-se oligossintomatico, apenas com desconforto abdominal leve. Não possuía histórico familiar para doenças neoplásicas, apenas hipertensão arterial sistêmica, fazendo tratamento para tal patologia com uso de antagonista do canal de cálcio, anlodipino, além de doença renal crônica não dialítica.

Trouxe consigo tomografia computadorizada (TC) de abdome evidenciando espessamento parietal concêntrico de aspecto infiltrativo em projeção do íleo distal/apêndice cecal com redução luminal importante, com apêndice difusamente espessado com perda difusa da definição de suas paredes, margeado por coleção líquida, inflamatória de paredes espessadas, medindo cerca de 6,1 x 5 x 4 (LL X AP X CC), com volume estimado em 63 mL, associada a intenso espessamento edematoso/inflamatório da gordura mesentérica adjacente (flegmão), mas sem determinar obstrução intestinal. Além disso, evidenciou espessamento duodenal da primeira e segunda porção. 
Realizou nova TC de abdome no serviço, com cerca de sete dias da primeira com achados compatíveis com apendicite complicada (rota), associada à coleção inflamatória adjacente com presença de plastrão, com apêndice difusamente espessado e perda da efinição de suas paredes, porém não foi mais evidenciada alteração duodenal, ainda assim foi solicitada esofagogastroduodenoscopia pela alteração sugerida no primeiro exame que evidenciou apenas gastrite enantematosa leve. TC de tórax com contraste evidenciou apenas espondiloartrose torácica. Foi iniciado esquema antibiótico de amplo espectro (ceftriaxona e metronidazol).

Paciente permaneceu internado por cerca de nove dias para realização de exames e seguimento clínico, evoluindo com melhora clínica importante, ausência de febre em todo internamento e eliminação da dor referida no início do quadro clínico, com exames físico e laboratoriais seriados dentro da normalidade, inclusive com marcadores tumorais, a não ser por creatinina que alcançou níveis de $2,44 \mathrm{mg} / \mathrm{dl}$ com clearence de creatinina estimado em $33,42 \mathrm{ml} / \mathrm{min}$ porém com melhora, chegando a $1,57 \mathrm{mg} / \mathrm{dl}$ e clearence estimado de 55.6 $\mathrm{ml} / \mathrm{min}$, recebendo alta hospitalar para conclusão de antibióticoterapia, programada para completar dez dias e seguimento ambulatorial semanal para programação de procedimento cirúrgico. Após cerca de sete dias foi readmitido no serviço pois apresentou quadro de dor semelhante ao quadro inicial, porém de resolução espontânea, permanecendo internado para dar seguimento do quadro com tratamento operatório cerca de quatro dias depois.

Optamos pela abordagem laparoscópica. Os achados cirúrgicos foram: distorção anatômica com dificuldade inicial para observação do ceco e apêndice, sendo necessário manobra de Cattell-Braasch com liberação parcial do cólon direito para melhor exposição do ceco e consequentemente apêndice, quando foi possível observar apêndice bloqueado em FID (fossa ilíaca direita) com aderência com sigmoide além de íleo terminal e plastrão com conteúdo purulento em cápsula fibrótica secundária ao bloqueio, apêndice vermiforme necrótico sem perfuração macroscópica e com base cecal integra, sendo abordado mesoapêndice, isolamento deste com descolamento das aderências, ligadura de sua base. $\mathrm{O}$ paciente evoluiu clinicamente bem, sem dor, náuseas ou outras queixas no pós operatório imediato e, recebendo alta hospitalar no dia seguinte.

Paciente compareceu para retorno ambulatorial com sete dias sem queixas álgicas, com ferida operatória sem sinais de complicações, para resgate do histopatológico que evidenciou periapendicite aguda sem sinais de malignidade. Seguimento com trinta e sessenta dias também sem alterações clínicas. 


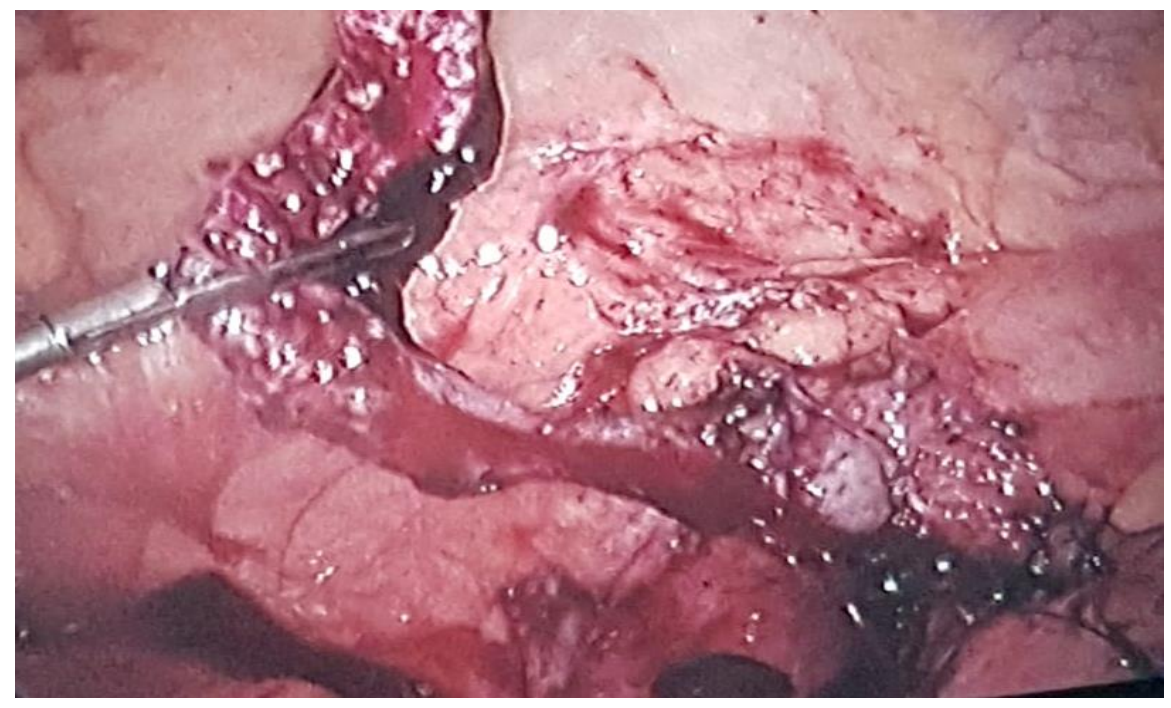

Figura 1 - Apêndice cecal dissecado com base íntegra.

\section{Discussão}

Em geral, existem três abordagens de tratamento para apendicite: a. abordagem "clássica" que compreende o manejo conservador (antibióticos e drenagem percutânea das coleções associadas) seguidas de intervalo de tempo até apendicectomia, b. apendicectomia imediata e c. abordagem totalmente conservadora que visa evitar completamente apendicectomia. (Forsyth et al., 2016; Taguchi et al., 2015)

Historicamente, a base da terapia para apendicite aguda consiste na cirurgia, com uma dada relevância para apendicectomia laparoscópica (AL) como o padrão-ouro de tratamento desde o início dos anos 90. Esforços recentes para diminuir os riscos associados à terapia cirúrgica da apendicite complicada incluem a introdução de manejo não operatório inicial com antibióticos intravenosos e apendicectomia com intervalo após a inflamação ter diminuído, proporcionando uma operação com menos complicações. Casos de apendicite com flegmão e abscesso são responsáveis por cerca de 2 a $10 \%$ de todos os casos de apendicite complicada. (Talan EA et al., 2017; Rashid et al., 2013)

Apendicectomia laparoscópica, realizada pela primeira vez por Semm em 1983, tornou-se recentemente uma abordagem cirúrgica bem aceita para o tratamento da apendicite complicada e foi idealizada por reduzir a duração da internação, otimizar o tempo de 
recuperação pós-operatória, produzir melhores resultados estéticos e mitigar a dor. No entanto, os autores de vários estudos em que a AL foi aplicada no tratamento de apendicite complicada alertam para o risco de infecção, com particular referência ao abscesso intraabdominal (AAI) e infecção superficial do sítio cirúrgico (ISC). Portanto, o uso de AL para apendicite complicada permaneceu um tema do debate. (Takami et al., 2019)

O tempo necessário para concluir uma apendicectomia aberta (AA) com intervalo foi significativamente menor que por via laparoscópica. Isso pode ser atribuído à curva de aprendizado da AL. Esta possui a vantagem inerente de inspecionar toda a cavidade abdominal e, portanto, pode diagnosticar qualquer patológica concomitante com mais frequência do que qualquer cirurgia aberta. Ademais, como a laparoscopia envolve pouca manipulação intestinal, espera-se que o retorno da atividade intestinal seja mais precoce. A duração do íleo foi significativamente menor. Observou-se uma diminuição no tempo de internação hospitalar bem como retomada precoce às atividades de vida cotidiana. Cumpre ressaltar que consiste numa opção é segura e eficaz. (Rashid et al., 2013; Takami et al., 2019)

Revisões anteriores avaliando com tentativas de tratamento conservador a fim de posterior abordagem laparoscópica detectaram o surgimento de complicações tais como falhas da drenagem, sintomas persistentes, abscessos recorrentes, e uma carga financeira pesada foram os desafios para implantação dessa estratégia terapêutica. (Al-Kurd et al., 2018) Contudo, um estudo prospectivo em 2010, considerado um dos pioneiros na investigação do tratamento laparoscópico de apendicite complicada concluiu que os resultados obtidos dependem da experiência da equipe cirúrgica bem como de recursos da instituição. (Peter et al., 2010)

O papel do tratamento antibiótico conservador na abordagem inicial da apendicite aguda não complicada é controversa. Devido a lacunas de evidência, não está recomendado o tratamento antibiótico rotineiramente a fim de substituir cirurgia. (Talan DA et al., 2018). Tipicamente, após o manejo não-operatório bem-sucedido de um flegmão, apendicectomia com intervalo é realizada semi-eletivamente ou eletivamente. A terapêutica conservadora é comum nesses casos, mas tem sido questionada por uma quantidade crescente de evidências (Talan EA et al., 2017. Outra questão importante e que ainda é motivo de debate, é o momento ideal para a apendicectomia após o tratamento conservador inicial. (Di Saverio et al., 2016; Rashid et al., 2013)

O objetivo de retardar apendicectomia nos casos que se apresentam com abscesso ou flegmão é diminuir a probabilidade de complicações, de fato percebeu-se tecidos menos 
friáveis à manipulação apesar da distorção anatômica encontrada no nosso caso. Essa abordagem apresentou taxas relativamente baixas $(<10 \%)$ de recorrência de apendicite ou abscesso após tratamento conservador inicial. Meta-análises constataram que tratamento conservador está associado com complicações semelhantes ou menores em comparação com cirurgia inicial e uma diretriz recente agora reconhece que essa modalidade pode ser bemsucedida para pacientes selecionados que desejam evitar a cirurgia precocemente (Talan DA et al., 2018).

As taxas de resposta a antibióticos em ensaios utilizando esses medicamentos foram altos, 88\% a 100\%, com duração de 7-10 dias. [Di Saverio et al., 2016; Talan DA et al., 2018) Essa abordagem não invasiva inicialmente decorre da preocupação devido a anatomia distorcida, podendo levar a lesões de estruturas intra-abdominais circunvizinhas e possíveis possibilidades de ressecção ileocólica ou hemicoletomia. (Al-Kurd et al., 2018)

Existem três razões principais para lançar mão de investigação complementar no contexto da massa do apêndice: em primeiro lugar avaliar a resposta da massa / abscesso ao tratamento conservador segundo, para verificar se existe algum fecalito, que alguns autores consideram como fator isolado de recorrência, e terceiro, para excluir outros diagnósticos. Orienta-se o acompanhamento com USG (ultrassonografia) / TC que devem ser realizadas após 4-6 semanas, além da colonoscopia, particularmente em adultos acima de 40 anos (Forsyth et al., 2016). Uma grande preocupação manifestada em alguns estudos em relação a apendicectomia com intervalo é adiamento do diagnóstico de neoplasia. (Cheng et al, 2017; Talan DA et al, 2018). Em nosso caso, justamente foi solicitada outra TC de abdome no intuito de afastar tal possibilidade, além de elucidar melhor a alteração duodenal encontrada no primeiro exame, realizado fora do nosso serviço.

Em ensaio prospectivo, o momento da abordagem para apendicectomia compreendeu cerca de 6-9 semanas após tratamento inicial, no presente estudo a abordagem cirúrgica ocorreu cerca sete semanas após o início dos sintomas tendo em vista o retorno do paciente ao serviço por recrudescência, mesmo que esporádica, do quadro álgico, compatível com os dados da literatrua que enfatizam a maior recorrências dos sintomas em torno de seis semanas. (Tanaka et al., 2016).

Não se encontraram dados que evidenciem diferenças na morbidade ou mortalidade entre o grupo da apendicectomia precoce e apendicectomia tardia (Talan EA et al., 2017). Além disso, o período de uso de analgésicos, a taxa de readmissão após a cirurgia e a ocorrência de complicações não foram estatisticamente diferentes entre os dois grupos, embora o tempo 
operatório e o período de jejum tendam a ser mais curtos nos pacientes submetidos à apendicectomia com intervalo, representando uma escolha superior para apendicite com abscesso ou fleuma bem circunscrito, nesse sentido. (Tanaka et al., 2016)

O advento da cirurgia laparoscópica levou à ideia de realizar apendicectomia também por esta via de acesso. A eficácia da abordagem laparoscópica para apendicite complicada tem sido extensivamente estudada. (Taguchi et al., 2015) Por conseguinte, muitos estudos foram realizados para comparar a laparoscopia com a apendicectomia aberta na apendicite aguda; no entanto, não existiam estudos prospectivos randomizados controlados comparando essas duas abordagens na apendicectomia com intervalo. Até então, havia estudos prospectivos de coorte e poucas revisões retrospectivas publicadas sobre a apendicectomia com intervalo. (Rashid et al., 2013) Recentemente, muitos estudos, estão advogando em favor da apendicectomia com intervalo com estratégia de escolha para apendicite complicada com abscesso circunscrito ou fleumão. (Rashid et al, 2013; Talan DA et al., 2018)

Com a publicação de evidências mais consistentes, a estratégia de tratamento conservador tornou-se estatisticamente significativa, permanecendo válida até agora. Contudo, a partir de 2010, após publicação de dois estudos clínicos randomizados, demonstrou-se o início da mudança de paradigma em favor da abordagem laparoscópica após intervalo de tratamento conservador, em casos selecionados (Gavriilidisa et al., 2019, Tanaka et al., 2016)) Defensores da apendicectomia laparoscópica afirmam que as vantagens do procedimento incluem melhor cicatrização de feridas, redução pós-operatória da dor e, finalmente, alta precoce do hospital, todos traduzindo para um retorno anterior à atividade normal. Eles também apoiam a ideia de avaliar laparoscopicamente a cavidade peritoneal antes de proceder a apendicectomia, particularmente em casos difíceis. (Taguchi et al., 2015; Rashid et al., 2013; Takami et al., 2019)

Três ensaios clínico de alta relevância e metanálise não expressaram nenhuma diferença significativa entre apendicectomia laparoscópica e tratamento conservador, muito embora, o efeito de ambos os estudos favoreceu o procedimento de apendicectomia laparoscópica inicial. Isso demonstra que o impacto do efeito desses ensaios clínicos randomizados sobre as evidências existentes impulsionam a mudança de paradigma que favorece a abordagem laparoscópica. (Gavriilidisa et al., 2019). Além disso, a eficácia da abordagem laparoscópica para apendicite complicada tem sido cada vez mais investigada. (Forsyth et al., 2016; Taguchi et al., 2015) 
Em síntese, a apendicectomia laparoscópica demonstrou menor tempo de internação, embora não tenha culminado numa melhor redução estatisticamente significativa da taxa de incidência de abscessos abdominais / pélvicos. (Gavriilidisa et al., 2019) As críticas à AL incluem: aumento do custo operatório, principalmente devido ao uso de instrumentos laparoscópicos descartáveis, aumento do tempo operatório e preocupações com maior incidência de abscessos intra-abdominais, principalmente após apendicite perfurada. (Rashid et al., 2013) Contudo, vários estudos mostraram que os principais benefícios da dessa abordagem para apendicite complicada incluem prevenção de infecção de ferida e duração reduzida da hospitalização. (Takami et al., 2019).

Constatou-se que a perda média de sangue foi significativamente menor no grupo AL do que no grupo AA. Isso é provável, porque aquela oferece um campo de visão cirúrgico melhor que o convencional, isso permite que os sangramentos sejam notados e tratados mais rapidamente com a abordagem laparoscópica. Vários estudos afirmam que a reintrodução de ingestão oral em AL ocorreu mais cedo. Vale ressaltar que o tempo de internação foi significativamente menor neste grupo. As taxas de complicações pós-operatórias bem como de infecção de ferida foram significativamente menor também. (Rashid et al., 2013; Takami et al., 2019)

As vantagens da AL já são consagradas na literatura em relação a AA, no entanto, o manejo da apendicite complicada permanece controverso em vários aspectos, dentre eles custos, período adequado para abordagem cirúrgica, seguimento adequado do paciente, dentre outros. Fatores que podem influenciar a abordagem poderia ser a infraestrutura local e os critérios para radiologia intervencionista para abscessos drenáveis; os conhecimentos e habilidades específicos necessários para o exame laparoscópico; e as preferências do cirurgião, com base na experiência. (Gavriilidisa et al., 2019).

\section{Conclusões}

A abordagem laparoscópica tem sido mais frequentemente empregada para apendicite complicada em adultos e crianças. Supressão da infecção de ferida operatória e redução do tempo de permanência hospitalar foram enfatizados como principais benefícios da AL para AA. Além disso, a apendicectomia laparoscópica está associada com redução nas taxas de dor pós-operatória com recuperação funcional mais rápida. Por outro lado, a existência de 
abscesso intra-abdominal continua sendo uma grande preocupação na AL. A apendicectomia com intervalo permanece um campo aberto a novos estudos uma vez que existem inúmeras lacunas ainda a serem preenchidas.

\section{Referências}

Al-Kurd A et al. (2018). Outcomes of interval appendectomy in comparison with appendectomy for acute Appendicitis. J Surg Res, Jeerusalem, n.225, p. 90-94.

Cheng Y, Xiong X, Lu J, Wu S, Zhou R, Cheng N. (2017). Early versus delayed appendicectomy for appendiceal phlegmon or abscess. Cochrane Libr, v. 6, p. 1-37.

Di Saverio S et al. (2016). WSES Jerusalem guidelines for diagnosis and treatment of acute appendicitis. World J Emerg Surg, EUA, v. 11, p.34.

Forsyth J, Lasithiotakis K, Peter M. (2016). The evolving management of the appendix mass in the era of laparoscopy and interventional radiology. Surgeon, EUA, v.15, n. 2, p. 109-115.

Gavriilidisa P, Angelisb N, Katsanos K, Saveriod S. (2019). Acute Appendicectomy or Conservative Treatment for Complicated Appendicitis (Phlegmon or Abscess)? A Systematic Review by Updated Traditional and Cumulative Meta-Analysis. J Clin Med Res, EUA, v. 11, n.1, p.56-64.

Peter S et al. (2010). Initial laparoscopic appendectomy versus initial nonoperative management and interval appendectomy for perforated appendicitis with abscess: a prospective, randomized trial. , EUA, v.45, n.1, p.236-40.

Rashid A et al. (2013). Laparoscopic Interval Appendectomy Versus Open Interval Appendectomy: A Prospective Randomized Controlled Trial. Surg Laparosc Endosc Percutan Tech, India, v. 23, n. 1, p. 93-96.

Taguchi Y et al. (2015). Laparoscopic versus open surgery for complicated appendicitis in adults: a randomized controlled Trial. Surg Endosc, Japan, p. 1-8.

Takami T et al. (2019). A clinical comparison of laparoscopic versus open appendectomy for the treatment of complicated appendicitis: historical cohort study. Eur J Trauma Emerg Surg, Japan, p. 1-5.

Talan DA, Saltzman DJ, DeUgarte, DA, Moran, J. (2018). Methods of conservative antibiotic treatment of acute uncomplicated appendicitis: A systematic review. J Trauma Acute Care Surg, Los Angeles, v.86, n. 4, p.722-736.

Talan EA et al. (2018). Operative versus non-operative therapy for acute phlegmon of the appendix: Is it afer? A case report and review of the literature. Int J Surg Case Rep, EUA, p.75-79. 
Tanaka Y et al. (2016). More than one-third of successfully nonoperatively treated patients with complicated appendicitis experienced recurrent appendicitis: Is interval appendectomy necessary? J Pediatr Surg, Japan, v.51, n. 12, p. 1957-1961.

\section{How to cite this article (APA format):}

Sá, George Wallisson Severo de; Batista, Hermes Melo Teixeira; Rolim, Francisco Felippe (2020). Current Approach to Acute Apendicitis: A Case Report. Am. In. Mult. J., Jul to Oct. (9) 5, 185-195.

Received: 09/04/2020;

Accepted: 09/18/2020. 